\title{
Juxtaglomerular cell tumor: A case report
}

\author{
HONGYUAN YANG ${ }^{*}$, ZUFEI WANG ${ }^{*}$ and JIANSONG JI \\ Department of Radiology, The Central Hospital of Lishui, Lishui, Zhejiang 323000, P.R. China
}

Received November 4, 2014; Accepted August 20, 2015

DOI: 10.3892/ol.2015.4041

\begin{abstract}
The current study reports the case of a 29-year-old female with a long-standing history of hypertension and headaches who presented to the Outpatient Clinic of The Central Hospital of Lishui (Lishui, Zhejiang, China). Abdominal ultrasound and contrast-enhanced computed tomography were performed, which showed a left renal neoplasm, prompting a diagnosis of renal angiomyolipoma or renal cell carcinoma. After a laparoscopic partial nephrectomy was performed, a number of different diagnoses were suggested by several pathologists from eight hospitals. Considering the patient's gender, age, medical history, histopathological features and immunohistochemistry, a final diagnosis of a juxtaglomerular cell tumor (JGCT) was established. The present study therefore indicates that the possibility of a JGCT should be considered when young adults present with renal parenchymatous tumors and high blood pressure. In addition, pathologists must take clinical information into account to form a precise diagnosis.
\end{abstract}

\section{Introduction}

A juxtaglomerular cell tumor (JGCT) is an extremely rare, benign renal neoplasm of myoendocrine cell origin (1) that was first described by Kihara et al in 1968 (2). To date, 100 cases of JGCT have been reported (3-13), however, few studies have systematically summarized its characteristic manifestations on computed tomography (CT) and magnetic resonance imaging (MRI) $(4,5)$. JGCT is characterized by renin production, hypokalemia and hypertension (5). Therefore, clinical features have assistant value for an accurate diagnosis. The diagnosis relies on pathological identification. The present study reports the case of a 29-year-old female who underwent a long process

Correspondence to: Dr Jiansong Ji, Department of Radiology, The Central Hospital of Lishui, 289 Kuocang Road, Liandu, Lishui, Zhejiang 323000, P.R. China

E-mail: jjstcty@163.com

${ }^{*}$ Contributed equally

Key words: juxtaglomerular cell tumor, computed tomography, pathology, diagnosis for the confirmation of a JGCT. Written informed consent was obtained from the patient.

\section{Case report}

A 29-year-old female presented to the Outpatient Clinic of The Central Hospital of Lishui (Lishui, Zhejiang, China) on April 15, 2013, due to headaches and hypertension.

The patient suffered from recurrent headaches and reported a two-year history of hypertension. The patient had previously been prescribed Norvasc, which effectively controlled the blood pressure. Furthermore, in April 2012, the patient was admitted to The First Affiliated Hospital of Zhejiang University (Hangzhou, China) with hypertension and subsequently underwent contrast-enhanced (CE)-CT, which found a left renal neoplasm. However, due to a lack of tumor-related symptoms, no further diagnosis was made and no treatment was provided.

Upon admission to The Central Hospital of Lishui in April 2013, physical examinations showed no abnormal findings, but hypokalemia was noted (potassium, $3.22 \mathrm{mmol} / \mathrm{l}$; normal range, 3.5-5.5 mmol/l). The patient's blood pressure was 140/100 $\mathrm{mmHg}$. Renal function, urinalysis, and other chemical and hematological profiles showed no abnormalities, with the exception of increased peripheral plasma renin activity (32 pg/ml/h; normal range, 0.3-2.9 pg/ml/h) and a high aldosterone level (324.65 pg/ml; normal range, 10-160 pg/ml).

Abdominal US revealed a low echo solid mass measuring $2.2 \times 1.8 \mathrm{~cm}$ in the left kidney. Unenhanced CT revealed a clearly demarcated isodensity lesion in the upper-middle region of the left kidney (Fig. 1). CE-CT demonstrated that the tumor was not markedly enhanced in the corticomedullary phase, but that it was further enhanced in the parenchymal phase and that the density slightly decreased in the excretory phase (Fig. 2). CT angiography (CTA) revealed the mass and normal renal vessels. The patient did not undergo MRI.

A retroperitoneoscopic left nephrectomy was performed on April30,2013.Grossly, a well-demarcated mass of 2.2x1.8x1.5 cm in size was located in the left kidney. Microscopic examination revealed that the tumor was composed of spindle cells (Fig. 3) with well-defined cell membranes. Polygonal cells (Fig. 4) with abundant eosinophilic cytoplasm, indistinct cell borders and nuclear atypia were observed (Fig. 5). Immunohistochemical findings: Vimentin (+), cluster of differentiation (CD)34 (++), B-cell lymphoma 2 (-/+), CD99 (-/+), CD117 (-/+), Dog-1 (-), human melanoma black 45 (-), Melan-A (-), S-100 (-), cytokeratin (-), epithelial membrane antigen $(-)$, Synaptophysin (Syn; +), 


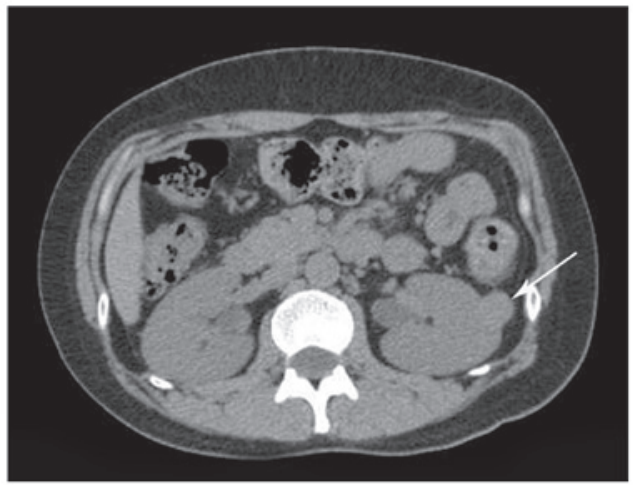

Figure 1. Unenhanced computed tomography scan revealing a well-defined isodensity mass of $2.2 \times 1.8 \mathrm{~cm}$ in size (arrow) in the left kidney.

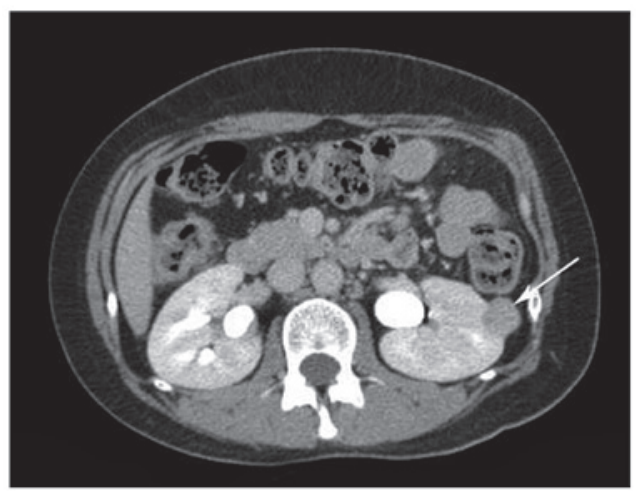

Figure 2. Enhanced computed tomography showing the tumor (arrow) as slightly less dense than the renal medulla (excretory phase).

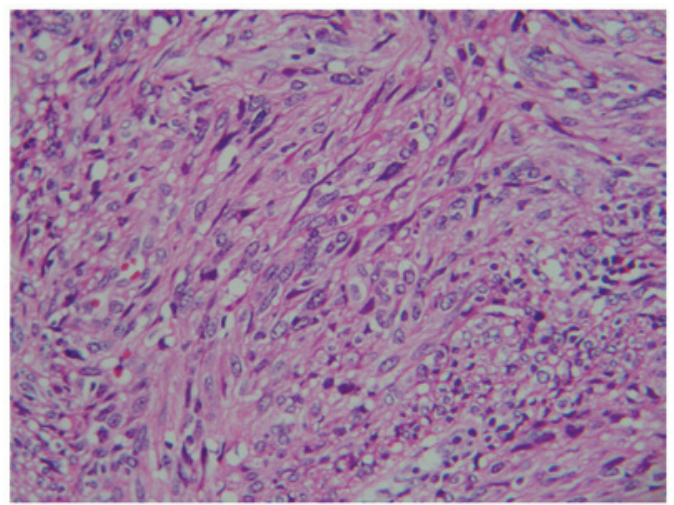

Figure 3. Spindle-shaped tumor cells (hematoxylin and eosin staining; magnification, $\mathrm{x} 200$ ).

CD56 (-), smooth muscle actin (-/+) and Desmin (-), with a Ki-67 of 6-8\%. The diagnosis was of a solitary fibrous tumor (SFT). Consultation with pathologists from other hospitals resulted in five pathologists agreeing with this diagnosis, while three other pathologists suggested a diagnosis of an undifferentiated sarcoma, sarcomatoid carcinoma or mesoblastic nephroma. Finally, the diagnosis of JGCT was formed due to the following factors: Young female patient; hypertension, hypokalemia and elevated plasma renin activity; histological morphology; positivity for CD34 and Syn; and post-operative normal blood pressure and plasma renin activity. The patient

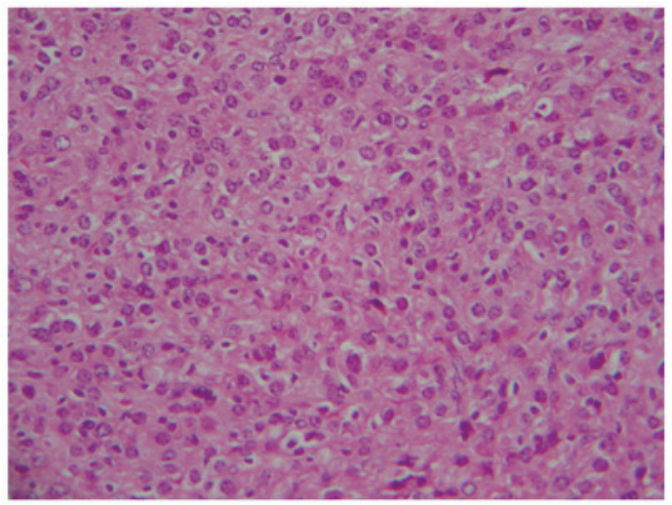

Figure 4. Polygonal cells with abundant eosinophilic cytoplasm (hematoxylin and eosin staining; magnification, $\mathrm{x} 200$ ).

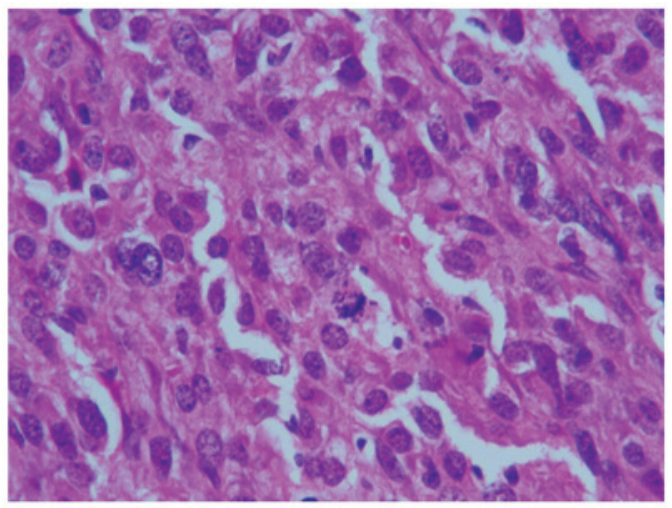

Figure 5. Tumor cells with a low degree of atypia and karyokinesis (hematoxylin and eosin staining; magnification, $\mathrm{x} 400$ ).

was followed up for 2 years and 6 months, with no evidence of tumor recurrence.

\section{Discussion}

JGCT mostly affects young adults. The peak age of incidence is in the second and third decades, with a 2:1 female preponderance (5). Although a JGCT is generally considered to be a benign tumor, one metastatic tumor has been reported (6). Therefore, an early diagnosis and surgery is necessary. In the present study, a case of a JGCT is reported and the significance of clinical characteristics for the pathological diagnosis of a JGCT is discussed.

The majority of JGCT patients exhibit a clinically typical presentation, including hypertension, hyperreninemia, hyperaldosteronism and hypokalemia (5). In the present study, hypertension was the first and most prominent manifestation, and the main reason for attendance at hospital, which is consistent with previously reported cases (7). Although one case of non-functioning JGCT has also been reported (8), the clinical features are still important for a definite diagnosis. No correlation has been found between the severity of symptoms and the size of the tumor (7).

Thus far, the US features of JGCT have not been reported. The majority of JGCTs reported in the literature are low- or isodensity, well-circumscribed, cortical tumors 
on plain CT scanning (7,9), which is consistent with the findings of the current study. Despite being hypervascular, the tumor appears hypovascular on CE-CT and CE-MRI, possibly due to renin-induced vasoconstriction (4). None of the reported JGCTs were stained during the corticomedullary phase, but all were stained moderately during the late phase following contrast enhancement. The imaging manifestations of JGCT are non-specific and indistinguishable from those of other solid renal neoplasms (5).

Histologically, JGCT consists of sheets of polygonal or spindle-shaped cells and a hemangiopericytic angioarchitecture (3). It is occasionally difficult to differentiate a JGCT from an SFT or MN $(3,10,11)$. Each of these three tumors is composed of spindle cells, and has a similar morphology and CD34 expression pattern. However, MNs are commonly found in infants aged $\leq 6$ months (11), and the patient in the present case was 29 years old. The majority of SFT patients have no clinical symptoms (10). Ultimately, by combining clinical symptoms, medical history and laboratory examination results, the definitive diagnosis of JGCT was established.

In conclusion, JGCT is a rare benign renal neoplasm. Only with sufficient expertise and integration with clinical characteristics can pathologists obtain evidence of a JGCT.

\section{Acknowledgements}

The authors would like to thank the associated staff from the First Affiliated Hospital of Zhejiang University (Hangzhou, Zhejiang, China), the Zhejiang Provincial People's Hospital (Hangzhou, Zhejiang, China), the Indiana University School of Medicine (Indianapolis, IN, USA) and the University of Massachusetts Medical School (Worcester, MA, USA) for their assistance with the pathological diagnosis.

\section{References}

1. Martin SA, Mynderse LA, Lager DJ and Cheville JC: Juxtaglomerular cell tumor: A clinicopathologic study of four cases and review of the literature. Am J Clin Pathol 116: 854-863, 2001.
2. Kihara I, Kitamura S, Hoshino T, Seida H and Watanabe T: A hitherto unreported vascular tumor of the kidney: A proposal of 'juxtaglomerular cell tumor'. Acta Pathol Jpn 18: 197-206, 1968.

3. Kuroda N, Maris S, Monzon FA, Tan PH, Thomas A, Petersson FB, Gatalica Z, Ghazalpour A, Bender RP, Grossmann P, et al: Juxtaglomerular cell tumor: A morphological, immunohistochemical and genetic study of six cases. Hum Pathol 44: 47-54, 2013.

4. Tanabe A, Naruse M, Ogawa T, Ito F, Takagi S, Takano K, Ohashi H, Tsuchiya K, Sone M, Nihei H and Toma H: Dynamic computer tomography is useful in the differential diagnosis of juxtaglomerular cell tumor and renal cell carcinoma. Hypertens Res 24: 331-336, 2001

5. Prasad SR, Surabhi VR, Menias CO, Raut AA and Chintapalli KN: Benign renal neoplasms in adults: Cross-sectional imaging findings. AJR Am J Roentgenol 190: 158-164, 2008

6. Duan X, Bruneval P, Hammadeh R, Fresco R, Eble JN, Clark JI, Vigneswaran WT, Flanigan RC and Picken MM: Metastatic juxtaglomerular cell tumor in a 52-year-old man. Am J Surg Pathol 28: 1098-1102, 2004.

7. Qiang J, Gao W, Chen D, Nie Z, Guan W, Li Y and Yu W: CT manifestations of renal juxtaglomerular cell tumor. Zhong Hua Fang She Xue Za Zhi 44: 885-886, 2010 (In Chinese).

8. Sakata R, Shimoyamada H, Yanagisawa M, Murakami T, Makiyama K, Nakaigawa N, Inayama Y, Ohashi K, Nagashima Y, Yao M and Kubota Y: Nonfunctioning juxtaglomerular cell tumor. Case Rep Pathol 2013: 973865, 2013.

9. Prasad SR, Narra VR, Shah R, Humphrey PA, Jagirdar J, Catena JR, Dalrymple NC and Siegel CL: Segmental disorders of the nephron: Histopathological and imaging perspective. $\mathrm{Br}$ J Radiol 80: 593-602, 2007.

10. Wang Z, Li K, Dong K, Liu G, Xiao X and Zheng S: Eight cases of congenital mesoblastic nephroma: Clinical analysis and literature review. Zhong Hua Xiao Er Wai Ke Za Zhi 34: 754-756, 2013 (In Chinese).

11. Lingying ZH, Enyu W, Suming $\mathrm{CH}$ and Linghui X: Solitary fibrous tumor of the kidney with duodenal stromal tumor: A case report. Zhong Hua Fang She Xue Za Zhi 41: 778, 2007 (In Chinese).

12. Elouazzani H, Jahid A, Bernoussi Z and Mahassini N: Juxtaglomerular cell tumor: A distinct mesenchymal tumor of kidney. J Clin Imaging Sci 4: 33, 2014.

13. Ren GP, Yu XR, Li YX, Wang LJ, Wang JQ, Shi HQ and Ye HH: Juxtaglomerular cell tumor of the kidney: A clinicopathologic analysis of five cases. Zhonghua Bing Li Xue Za Zhi 32: 511-515, 2003. 